\title{
Prescribing of opioid analgesics and related mortality before and after the introduction of long-acting oxycodone
}

\author{
Irfan A. Dhalla MD MSc, Muhammad M. Mamdani PharmD MPH, Marco L.A. Sivilotti MD MSc, \\ Alex Kopp BA, Omar Qureshi MD, David N. Juurlink MD PhD
}

Previously published at www.cmaj.ca

$\infty \infty$

See related commentary by Fischer and Rehm, page 881

\section{ABSTRACT \\ Introduction: Opioid-related mortality appears to be increas- ing in Canada. We examined the true extent of the problem and the impact of the introduction of long-acting oxycodone.}

Methods: We examined trends in the prescribing of opioid analgesics in the province of Ontario from 1991 to 2007. We reviewed all deaths related to opioid use between 1991 and 2004. We linked 3271 of these deaths to administrative data to examine the patients' use of health care services before death. Using time-series analysis, we determined whether the addition of long-acting oxycodone to the provincial drug formulary in January 2000 was associated with an increase in opioid-related mortality.

Results: From 1991 to 2007, annual prescriptions for opioids increased from 458 to 591 per 1000 individuals. Opioidrelated deaths doubled, from 13.7 per million in 1991 to 27.2 per million in 2004. Prescriptions of oxycodone increased by $850 \%$ between 1991 and 2007. The addition of long-acting oxycodone to the drug formulary was associated with a 5fold increase in oxycodone-related mortality $(p<0.01)$ and a $41 \%$ increase in overall opioid-related mortality $(p=0.02)$. The manner of death was deemed unintentional by the coroner in $54.2 \%$ and undetermined in $21.9 \%$ of cases. Use of health care services in the month before death was common: for example, of the 3066 patients for whom data on physician visits were available, $66.4 \%$ had visited a physician in the month before death; of the 1095 patients for whom individual-level prescribing data were available, $56.1 \%$ had filled a prescription for an opioid in the month before death.

Interpretation: Opioid-related deaths in Ontario have increased markedly since 1991. A significant portion of the increase was associated with the addition of long-acting oxycodone to the provincial drug formulary. Most of the deaths were deemed unintentional. The frequency of visits to a physician and prescriptions for opioids in the month before death suggests a missed opportunity for prevention.

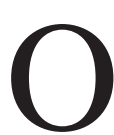
pioid analgesics are among the most commonly prescribed medications in Canada. One reason for their popularity is an increased awareness that these drugs may be appropriate for patients with chronic nonmalignant pain. ${ }^{1}$ Coincident with the increased willingness of physicians to prescribe opioids are reports of increasing opioid abuse and opioid-related deaths..$^{2-9}$ Particular attention has been focused on a long-acting formulation of oxycodone (OxyContin, Purdue Pharma) approved by Health Canada in 1996 and added to the Ontario provincial drug formulary in 2000. ${ }^{10}$ Oxycodone is a potent agonist of the mu- and kappa-opioid receptors, with an analgesic potency greater than that of morphine. ${ }^{11}$ Although the original product monograph stated that the long-acting formulation would impart a lower risk for abuse compared with other opioid analgesics, it was quickly recognized that the product's controlled-release characteristics could be defeated simply by chewing or crushing the tablets. ${ }^{12}$

We performed a study to determine whether opioid-related mortality increased following the introduction of long-acting oxycodone. We analyzed trends in the prescribing of opioids in Ontario between 1991 and 2007. We reviewed all deaths related to opioid use in Ontario between 1991 and 2004. We also examined the use of health care services before death among individuals whose deaths were related to opioid use.

\section{Methods}

\section{Prescribing data}

We obtained aggregated prescribing data from IMS Health Canada, which collects monthly prescription records from nearly two-thirds of all Canadian pharmacies. These data are extrapolated to provincial and national levels and are regularly used to characterize prescribing trends. ${ }^{13,14}$ Annual data on prescriptions for each opioid formulation were available from 1991 to 2001; detailed monthly data were available from 2001 to 2007. We included data on all opioid-containing analgesics and cough suppressants prescribed on an outpatient basis.

From the Departments of Medicine (Dhalla, Juurlink) and of Health Policy, Management and Evaluation (Dhalla), University of Toronto, Toronto, Ont.; the Department of Medicine and the Keenan Research Centre of the Li Ka Shing Knowledge Institute (Dhalla, Mamdani), St. Michael's Hospital, Toronto, Ont.; the Institute for Clinical Evaluative Sciences (Mamdani, Kopp, Juurlink), Toronto, Ont.; the Departments of Emergency Medicine and of Pharmacology and Toxicology (Sivilotti), Queen's University, Kingston, Ont.; the Department of Family Medicine (Qureshi), State University of New York at Buffalo, Buffalo, USA; Sunnybrook Health Sciences Centre (Juurlink), Toronto, Ont.; and the Faculty of Medicine (Mandani), King Saud University, Riyadh, Saudi Arabia

Cite as CMAJ 2009. DOI:10.1503/cmaj.090784 


\section{Deaths related to opioid use}

We examined the records of the Office of the Chief Coroner of Ontario to identify deaths related to opioid use. In accordance with Ontario's Coroners Act, all deaths that are sudden and unexpected, or unnatural, must be reported to the coroner's office. The coroner ascertains the cause and manner of death and, when necessary, conducts a postmortem examination that usually includes detailed toxicologic testing. We reviewed all deaths involving drugs or alcohol over the 14year period from Jan. 1, 1991, to Dec. 31, 2004. We did not analyze files from subsequent years because many later cases were still under review by the coroner at the start of our study. One of us (O.Q.) abstracted data using an encrypted computer database.

Deaths were deemed to have involved opioids if the coroner's investigation concluded so. In almost all instances, this conclusion was based on (a) a toxicologic analysis revealing that opioid concentrations were sufficiently high to cause death or (b) the coroner's determination that a combination of drugs (including at least one opioid present at a clinically significant concentration) resulted in death. Deaths were considered not to be related to opioid use if another drug was present at a concentration high enough to cause death and one or more opioids were present at levels that could be associated with therapeutic use. If the abstractor was uncertain about the cause of death, the file was reviewed by one or more of us (I.A.D., M.L.A.S. and D.N.J.) until consensus was achieved.

Before the collection of data began, a training exercise was performed in which the abstractor and the principal investigator independently abstracted data from 20 charts. Midway through the data-collection period, the validation exercise was repeated on another 20 charts. In both exercises, there was complete agreement on all major data fields, including whether or not each death was related to opioid use.

\section{Use of health care services before death}

To examine the use of health care services before death among individuals who died of opioid-related causes, we linked coroner's records with population-based health care data held at the Institute for Clinical Evaluative Sciences. ${ }^{15,16}$ Because complete data on health care utilization have been available as of July 1991, we included deaths that occurred on or after July 1, 1992, thereby allowing a full year of antemortem health care data. For a subset of patients with available data, we also analyzed patient-level use of prescription drugs in the year before death. In Ontario, complete prescription records are available from April 1991 onward for people 65 years or older, and from April 1997 onward for younger people who are covered under the public drug plan.

\section{Statistical analysis}

We performed descriptive analyses using population data from Statistics Canada $^{17}$ to standardize death rates and prescription rates. We used the number of opioid prescriptions dispensed from 1991 to 2007 as a proxy for the total quantity of opioids prescribed in Ontario. We validated this assumption for oxycodone specifically from 2001 to 2007, when more detailed prescribing data were available. For the people included in the analysis of health care utilization, we determined the median number of physician visits, emergency department visits and opioid prescriptions in the year before death. We also examined the time between the last physician visit and death, and the reason for the visit.

We used time-series analysis to examine temporal trends in oxycodone-related and opioid-related mortality. ${ }^{18} \mathrm{We}$ assessed the effect of the introduction of long-acting oxycodone to the drug formulary by using interventional autoregressive integrated moving average (ARIMA) models with ramp functions. Ramp functions are used to model the situation that occurs in a data series when there appears to be a change in the slope. ${ }^{19}$ To test

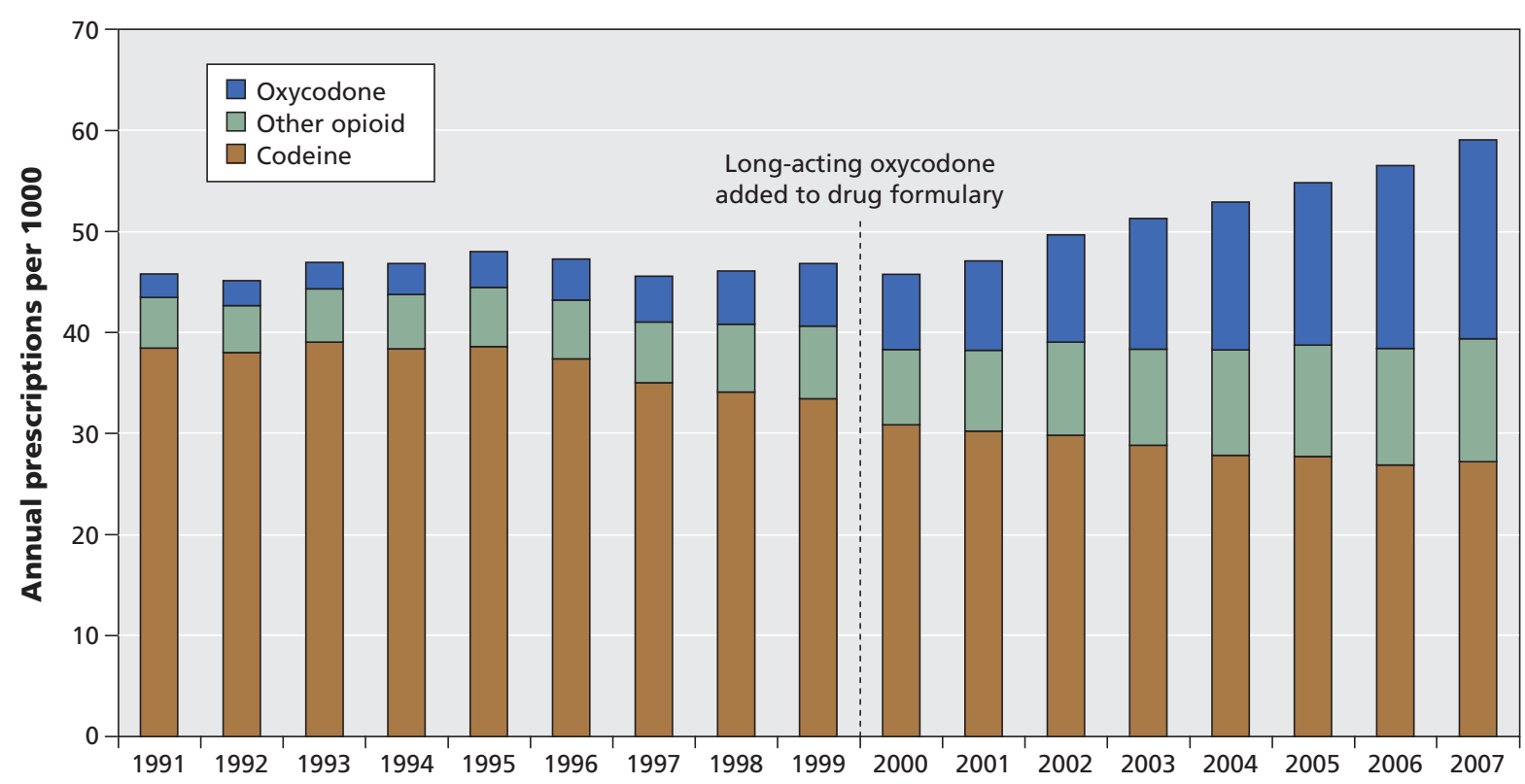

Figure 1: Annual number of opioid analgesics prescribed on an outpatient basis in Ontario from 1991 to 2007. 
the appropriateness of model assumptions, we assessed the autocorrelation, partial autocorrelation and inverse autocorrelation functions; performed the augmented Dickey-Fuller test $;^{20}$ and calculated the Ljung-Box $\chi^{2}$ statistic at various lags. ${ }^{21} \mathrm{We}$ adjusted statistical analyses for incomplete data in 1993 using a step function. All $p$ values were two-sided; we used a value of 0.05 as the threshold for statistical significance.

\section{Ethics approval}

This study was approved by the Research Ethics Board of the Sunnybrook Health Sciences Centre.

\section{Results}

\section{Prescribing of opioid analgesics}

From January 1991 to May 2007, the prescribing of opioid analgesics in Ontario increased by $29 \%$, from 458 to 591 prescriptions per 1000 individuals annually (Figure 1). Codeine was the most frequently prescribed agent, although the number of prescriptions for the drug declined gradually during the study period. In contrast, the number of oxycodone prescriptions rose more than $850 \%$ during the same period, from 23 per 1000 individuals in 1991 to 197 per 1000 in 2007. The prescribing of hydromorphone, fentanyl and morphine also increased considerably over the same period, although the total number of prescriptions for these drugs was far lower than that for oxycodonecontaining products (Figure 1). By 2006, oxycodone accounted for $32 \%$ of the almost 7.2 million prescriptions for opioids dispensed that year. Of the 2.3 million oxycodone prescriptions dispensed in 2006, 28\% were for the long-acting formulation.

From January 2001 to May 2007, the period during which we had detailed population-level prescribing data, the average amount of long-acting oxycodone dispensed per prescription increased by $24 \%$, from $1830 \mathrm{mg}$ to 2280 $\mathrm{mg}$. The amount of long-acting morphine dispensed per prescription increased by 3\%. During this period, the correlation between prescription counts of oxycodone and total mass of drug dispensed was exceptionally high $(r=0.996)$, which validates the use of prescription counts as a measure of prescribing before 2001 .

\section{Opioid-related deaths}

Between 1991 and 2004, the Office of the Chief Coroner of Ontario classified 7353 deaths as involving alcohol, drugs or both. Of these, the files of 254 individuals (3.5\%) were unavailable for study; approximately 180 of the missing files were for deaths during 1993. We examined the remaining 7099 records individually. In 3406 of these, opioids were implicated as a cause of death (Table 1).

Most of the 3406 deaths (2108 [61.9\%]) involved a single opioid (or possibly morphine and heroin together, since morphine is the only detectable metabolite in many heroin-related deaths). Morphine or heroin (or both) were involved in 761 of these deaths, of which 49 were confirmed to involve heroin based on the presence of the 6-monoacetylmorphine metabolite. The next most common opioids involved in single-opioid deaths were codeine $(n=431)$, methadone $(n=304)$ and oxycodone $(n=234)$.

\begin{tabular}{|c|c|}
\hline Characteristic & $\begin{array}{c}\text { No. }(\%) \\
\text { of people* }\end{array}$ \\
\hline Age, yr, median (IQR) $†$ & $40(34-48)$ \\
\hline Sex, malet & $2179(66.6)$ \\
\hline \multicolumn{2}{|l|}{ Manner of death } \\
\hline Unintentional & $1847(54.2)$ \\
\hline Suicide & $803(23.6)$ \\
\hline Undetermined & 745 (21.9) \\
\hline Other or missing data & $11(0.3)$ \\
\hline Single opioid associated with death $\ddagger$ & $2108(61.9)$ \\
\hline Morphine or heroin (or both)§ & $761(36.1)$ \\
\hline Codeine & 431 (20.4) \\
\hline Methadone & $304(14.4)$ \\
\hline Oxycodone & $234(11.1)$ \\
\hline Fentanyl & $57 \quad(2.7)$ \\
\hline Other & $321(15.2)$ \\
\hline
\end{tabular}

Note: IQR = interquartile range.

*Unless stated otherwise.

tValues for age and sex are based on the 3271 individuals whose files were linked to data on use of health care services.

¥The remaining 1298 deaths were either associated with a combination of opioids ( $n=1251$ ) or the specific opioid(s) could not be determined $(n=47)$.

$\S M o r p h i n e$ is the only detectable metabolite in many heroin-related deaths.

The majority of opioid-related deaths also involved at least 1 non-opioid central nervous system depressant. This was the case in $425(91.6 \%)$ of the 464 deaths involving oxycodone, for example. The most commonly implicated non-opioid central nervous system depressants in deaths related to oxycodone use were benzodiazepines ( $n=276$ [59.5\%]), alcohol (202 [43.5\%]) and cyclic antidepressants $(n=122$ [26.3\%]).

Opioid-related mortality doubled, from 13.7 per million in 1991 to 27.2 per million in 2004. The median age at death was 40 years (interquartile range $34-48$ years); $67 \%$ of the deaths occurred in men. The manner of death was deemed by the coroner to be unintentional in 1847 cases $(52.4 \%)$, suicide in $803(23.6 \%)$ and undetermined in $745(21.9 \%)$.

We observed a substantial increase in overall opioidrelated mortality following the addition of long-acting oxycodone to the provincial drug formulary in January 2000. Between 1999 and 2004, the annual number of opioid-related deaths increased by $41 \%(p=0.02)$, from 19.4 to 27.2 per million annually (Figure 2, top panel). The number of oxycodone-related deaths increased by $416 \%(p<0.01)$ during the same period, from 1.39 per million to 7.17 per million annually (Figure 2, bottom panel). The rise in opioid-related deaths was due in large part to inadvertent toxicity; there was no significant increase in the number of deaths from suicide involving opioids over the study period (Figure 3 ).

\section{Health care utilization before death}

We linked coroner's data to existing health care databases for $3271(96.0 \%)$ individuals who died of opioid-related causes. We included 3066 deaths that occurred on or after July 1, 
1992. Most of the patients (2037 [66.4\%]) were seen by a physician in an outpatient setting at least once in the 4 weeks before death. The median number of outpatient physician visits per individual in the 52 weeks before death was 15 (interquartile range 6-29). Emergency department visits were also common, with 1807 (58.9\%) of the individuals having at least 1 visit in the year before death.

The final encounter with a physician before death was an office visit for 2476 patients and an emergency department visit for 378 patients. The median number of days (and interquartile range) between the visit and death was 11 (4-34) for those who made an office visit and 9 (2-37) for those who went to an emergency department. Analysis of physician claims for these visits revealed that diagnoses of mental health problems (e.g., anxiety, depression or drug dependence) and pain-related complaints (e.g., joint pain or back pain) comprised the most common reasons for medical attention, which suggests that opioids prescribed to patients with these conditions may have contributed to death.

Of the 1095 patients for whom we had individual-level prescribing data, $897(81.9 \%)$ received at least 1 prescription for an opioid in the year before death; $614(56.1 \%)$ received at
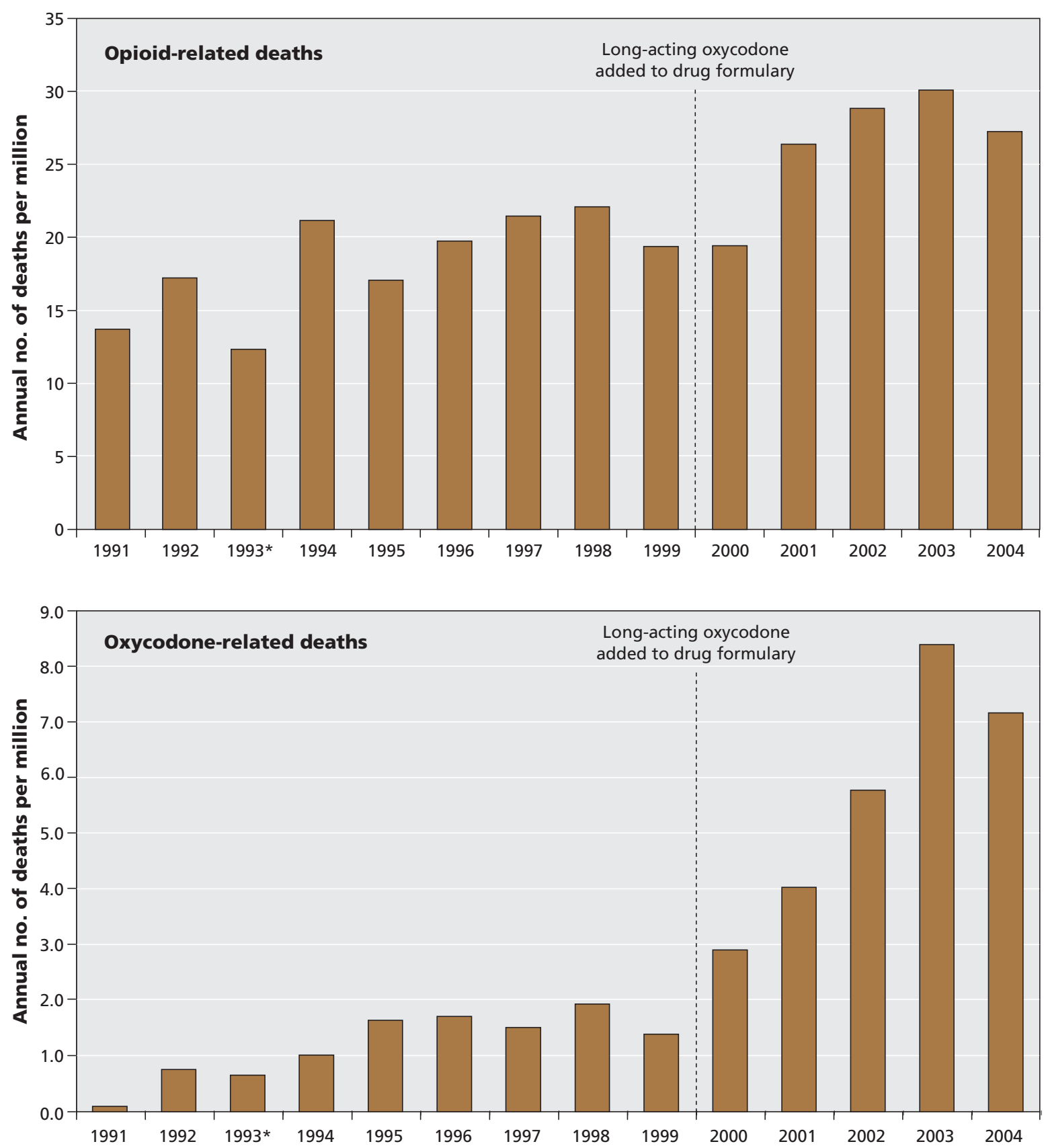

Figure 2: Deaths related to the use of opioid analgesics (top) and long-acting oxycodone (bottom) in Ontario, 1991 to $2004 .{ }^{*}$ The value for 1993 is an underestimate owing to missing data (see text for details). 
least 1 opioid prescription in the month before death. The median number of opioid prescriptions dispensed in the year before death was 10 (interquartile range 2-28). Many patients who were dispensed an opioid or other central nervous system depressant following their final physician visit had the same pharmaceutical identified on post-mortem toxicological analysis. For example, of the 75 individuals who were dispensed oxycodone following their last physician visit, 50 (66.7\%) had oxycodone present on post-mortem toxicological analysis.

\section{Interpretation}

We identified substantial increases in the prescribing of opioids and opioid-related mortality in Ontario over more than a decade. The prescribing of oxycodone rose far more rapidly than that of any other opioid. This was accompanied by a 5fold rise in oxycodone-related deaths, most of which were unintentional and involved patients taking other central nervous system depressants. We also observed an increase in overall opioid-related mortality following the addition of long-acting oxycodone to the provincial drug formulary. Finally, we found that use of health care services was high among individuals whose deaths were related to opioid use, with almost two-thirds having seen a physician in the month before death.

The societal burden of opioid-related mortality and morbidity in Canada is substantial. In our study, the annual incidence of opioid-related deaths in 2004 (27.2 per million) falls between the incidence of death from HIV infection (12 per million) and sepsis (40 per million). ${ }^{22}$ Moreover, with a median age of 40 years at death, the potential years of life lost from opioid-related death is almost certainly greater than that of many diseases primarily affecting older individuals. Although increases in opioid abuse and opioid-related deaths have been documented in several countries, ${ }^{6,723}$ some authors have argued that prescribing is not a major contributor to the adverse health consequences related to abuse of opioid analgesics. ${ }^{24,25}$ In contrast, our individual-level analysis strongly supports the hypothesis that increased rates of opioid prescribing contribute significantly to morbidity and unintentional opioid-related death.

As far as we are aware, there is no evidence that longacting oxycodone is more addictive or intrinsically more dangerous than other highly potent opioid analgesics. However, the marketing campaign that accompanied the introduction of long-acting oxycodone may have contributed to it being prescribed more liberally than other highly potent opioids. Notably, senior executives of Purdue Pharma pleaded guilty to charges of deceptive marketing in the United States and agreed to individual and corporate fines in excess of US\$600 million in 2007. ${ }^{26}$

In addition to a general increase in awareness, several specific interventions may help reduce the adverse health consequences of opioid prescribing. Real-time electronic databases accessible to physicians and pharmacists would make it more difficult for individuals to surreptitiously obtain opioids from multiple prescribers or pharmacies ${ }^{30}$ and might reduce the risk of drug interactions between opioids and other central nervous system depressants. ${ }^{31}$ Prescribers and pharmacists need to be better educated about the relative potency of different opioids, the use of physician-patient contracts in opioid prescribing, ${ }^{32-34}$ the outpatient care of opioid-dependent individuals $\mathrm{s}^{35,36}$ and the potential interaction of opioids with other central nervous system depressants. In some instances, physicians could use urine toxicology assays when prescribing opioids to help identify illicit drug use and drug diversion.$^{37}$ Finally, formulary restrictions could be used to deter the prescribing of pharmaceuticals with a high potential for abuse..$^{38-40}$

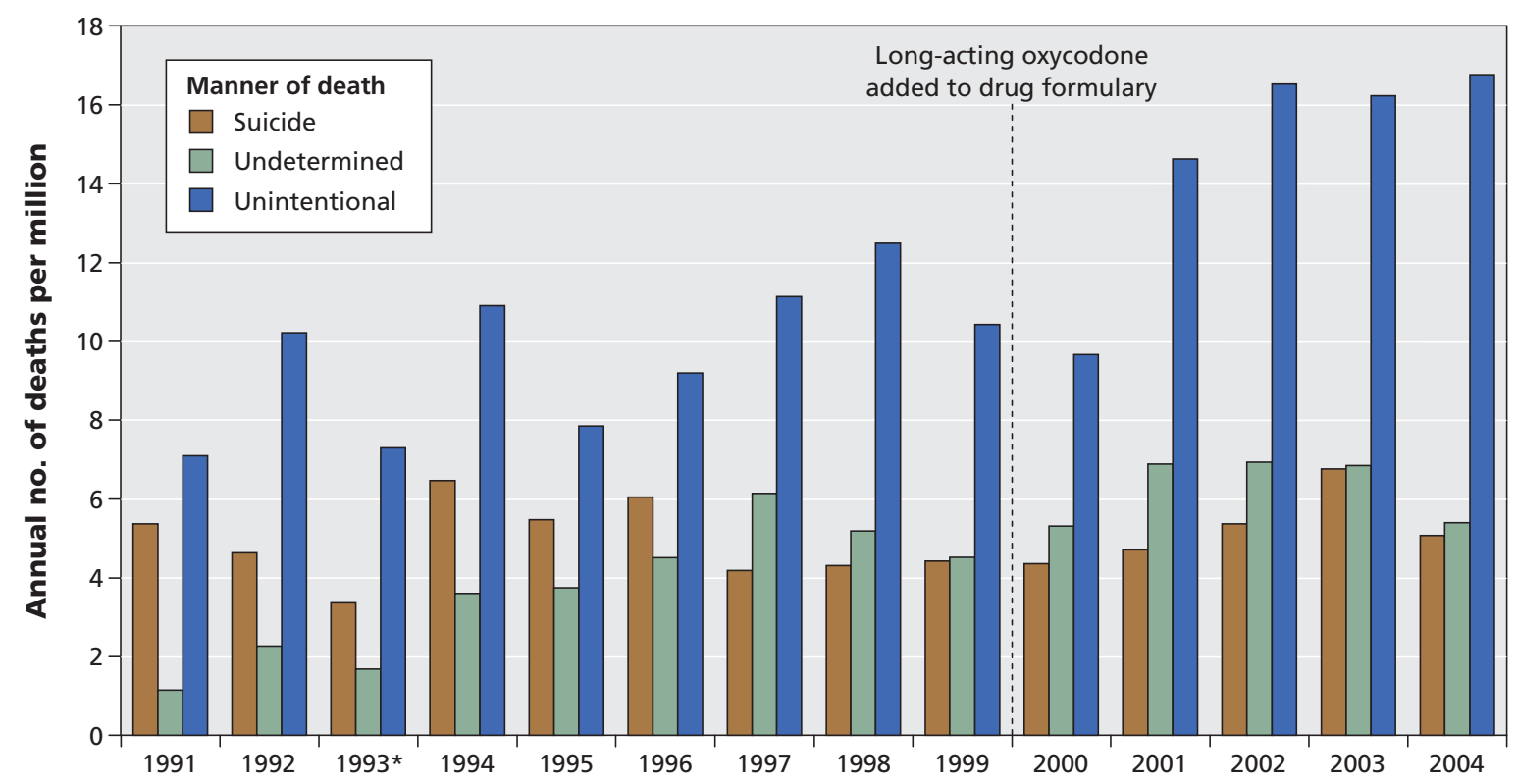

Figure 3: Deaths related to the use of opioid analgesics from 1991 to 2004 , by manner of death. *The value for 1993 is an underestimate owing to missing data. (See text for details.) 


\section{Limitations}

Our study has several limitations. First, because coroners do not investigate all deaths, particularly those involving elderly people, we may not have been able to account for all opioidrelated deaths. However, this limitation can only underestimate the true number of opioid-related deaths. Second, we did not have detailed data regarding opioid-related deaths after 2004. However, preliminary data from the Chief Coroner of Ontario suggest that the rates of oxycodone-related deaths and opioid-related deaths in Ontario have both continued to increase since 2004 (Dr. Andrew McCallum: personal communication, 2009). Finally, we relied on administrative data to assess use of health care services and prescribing. These data are incomplete and less detailed than individual health records. However, the methods and data used in our study are reliable and have been validated extensively. ${ }^{27-29}$

\section{Conclusion}

We found large and sustained increases in opioid prescribing and opioid-related mortality in Ontario over an extended period. The addition of long-acting oxycodone to the provincial drug formulary was associated with a 5-fold rise in deaths related to oxycodone use, as well as an increase in overall opioid-related mortality. The frequency of visits to a physician and prescriptions for opioids in the month before death suggests a missed opportunity for prevention.

This article has been peer reviewed.

\section{Competing interests: None declared.}

Contributors: Irfan Dhalla, Muhammad Mamdani, Marco Sivilotti and David Juurlink designed the study. Omar Qureshi was primarily responsible for the acquisition of data. Alex Kopp performed the statistical analyses. All of the authors contributed to the interpretation of the data. Irfan Dhalla prepared the initial draft of the manuscript. All of the authors critically revised the manuscript and approved the final version submitted for publication.

Acknowledgments: The authors are grateful to IMS Health Canada for providing prescribing data, Dr. Andrew McCallum, June Lindsell and Kathy McKague at the Office of the Chief Coroner of Ontario for project facilitation, assistance with data retrieval and help interpreting toxicologic analyses, and Lina Paolucci, Ashif Kachra and Patricia Parra for administrative assistance.

Funding: This work was supported by a research grant from the Canadian Institutes of Health Research. Irfan Dhalla was supported by a Fellowship Award, and David Juurlink a New Investigator Award, from the Canadian Institutes of Health Research. The Institute for Clinical Evaluative Sciences is funded by an annual grant from the Ontario Ministry of Health and LongTerm Care. The opinions, results and conclusions reported in this article are those of the authors and are independent from the funding sources. No endorsement by the Institute for Clinical Evaluative Sciences or the Ontario Ministry of Health and Long-Term Care is intended or should be inferred.

\section{REFERENCES}

1. Practice guidelines for chronic pain management. A report by the American Society of Anesthesiologists Task Force on Pain Management, Chronic Pain Section. Anesthesiology 1997;86:995-1004.

2. Cicero TJ, Inciardi JA, Munoz A. Trends in abuse of OxyContin and other opioid analgesics in the United States: 2002-2004. J Pain 2005;6:662-72.

3. Friedman RA. The changing face of teenage drug abuse - the trend toward prescription drugs. N Engl J Med 2006;354:1448-50.

4. Gilson AM, Ryan KM, Joranson DE, et al. A reassessment of trends in the medical use and abuse of opioid analgesics and implications for diversion control: 19972002. J Pain Symptom Manage 2004;28:176-88.

5. Increase in poisoning deaths caused by non-illicit drugs — Utah, 1991-2003. MMWR Morb Mortal Wkly Rep 2005;54:33-6.
6. Paulozzi LJ, Budnitz DS, Xi Y. Increasing deaths from opioid analgesics in the United States. Pharmacoepidemiol Drug Saf 2006;15:618-27.

7. Hall W, Lynskey M, Degenhardt L. Trends in opiate-related deaths in the United Kingdom and Australia, 1985-1995. Drug Alcohol Depend 2000;57:247-54.

8. Fischer B, Rehm J, Patra J, et al. Changes in illicit opioid use across Canada. CMAJ 2006;175:1385-7.

9. Fischer B, Rehm J, Goldman B, et al. Non-medical use of prescription opioids and public health in Canada: an urgent call for research and interventions development. Can J Public Health 2008;99:182-4.

10. Government accounting office. OxyContin abuse and diversion and efforts to address the problem: highlights of a government report. J Pain Palliat Care Pharmacother 2004;18:109-13.

11. Lugo RA, Kern SE. The pharmacokinetics of oxycodone. J Pain Palliat Care Pharmacother 2004;18:17-30.

12. Lipman AG. What have we learned from OxyContin? J Pain Palliat Care Pharmacother 2003; 17:1-4.

13. Jackevicius CA, Tu JV, Ross JS, et al. Use of ezetimibe in the United States and Canada. N Engl J Med 2008;358:1819-28.

14. Majumdar SR, McAlister FA, Soumerai S. Synergy between publication and promotion: comparing adoption of new evidence in Canada and the United States. Am J Med 2003;115:467-72.

15. Juurlink DN, Herrmann N, Szalai JP, et al. Medical illness and the risk of suicide in the elderly. Arch Intern Med 2004;164:1179-84.

16. Juurlink DN, Mamdani MM, Kopp A, et al. The risk of suicide with selective serotonin reuptake inhibitors in the elderly. Am J Psychiatry 2006;163:813-21.

17. Annual Demographic Statistics, 2005. Ottawa (ON): Statistics Canada; 2006. Cat. no. 91-213-XB.

18. Juurlink DN, Mamdani MM, Lee DS, et al. Rates of hyperkalemia after publication of the Randomized Aldactone Evaluation Study. N Engl J Med 2004;351:543-51.

19. Helfenstein U. Box-Jenkins modelling in medical research. Stat Methods Med Res 1996;5:3-22.

20. Dickey DA, Fuller WA. Distribution of the estimators for autoregressive time series with a unit root. J Am Stat Assoc 1979;74:427-31.

21. Ljung GM, Box GEP. On a measure of lack of fit in time series models. Biometrika 1978;65:297-303.

22. Age-standardized mortality rates by selected causes, by sex. Ottawa (ON): Statistics Canada; 2009. Cat. no. 84F0209X. Available: www40.statcan.gc.ca/101/cst01 /health30a-eng.htm (accessed 2009 Sept. 15).

23. Paulozzi LJ, Ryan GW. Opioid analgesics and rates of fatal drug poisoning in the United States. Am J Prev Med 2006;31:506-11.

24. Nicholson B. Responsible prescribing of opioids for the management of chronic pain. Drugs 2003;63:17-32.

25. Joranson DE, Ryan KM, Gilson AM, et al. Trends in medical use and abuse of opioid analgesics. JAMA 2000;283:1710-4.

26. Meier B. Narcotic maker guilty of deceit over marketing. New York Times 2007 May 11; Sect 1:1.

27. Levy AR, O'Brien BJ, Sellors C, et al. Coding accuracy of administrative drug claims in the Ontario Drug Benefit database. Can J Clin Pharmacol 2003;10:67-71.

28. Karen T, Norman RC, Xhong-Liang C, et al. Accuracy of administrative databases in identifying patients with hypertension. Open Medicine 2007;1:2007.

29. Shah BR, Hux JE, Laupacis A, et al. Administrative data algorithms can describe ambulatory physician utilization. Health Serv Res 2007;42:1783-96.

30. Fishman SM, Papazian JS, Gonzalez S, et al. Regulating opioid prescribing through prescription monitoring programs: balancing drug diversion and treatment of pain. Pain Med 2004;5:309-24.

31. Pharmanet. Victoria (BC): Ministry of Health Services, Government of British Columbia; 2007. Available: www.health.gov.bc.ca/pharmacare/pharmanet /netindex.html\# (accessed 2009 Sept. 15).

32. Hariharan J, Lamb GC, Neuner JM. Long-term opioid contract use for chronic pain management in primary care practice. A five year experience. J Gen Intern Med 2007;22:485-90

33. Wallace LS, Keenum AJ, Roskos SE, et al. Development and validation of a lowliteracy opioid contract. J Pain 2007;8:759-66.

34. Arnold RM, Han PK, Seltzer D. Opioid contracts in chronic nonmalignant pain management: objectives and uncertainties. Am J Med 2006;119:292-6.

35. Sullivan LE, Fiellin DA. Narrative Review: Buprenorphine for Opioid-Dependent Patients in Office Practice. Ann Intern Med 2008;148:662-70.

36. Fiellin DA, O'Connor PG. Office-Based Treatment of Opioid-Dependent Patients. N Engl J Med 2002;347:817-23.

37. Katz N, Fanciullo GJ. Role of urine toxicology testing in the management of chronic opioid therapy. Clin J Pain 2002;18(Suppl):S76-82

38. Huskamp HA, Deverka PA, Epstein AM, et al. The effect of incentive-based formularies on prescription-drug utilization and spending. N Engl J Med 2003;349:2224-32.

39. Simon GE, Psaty BM, Hrachovec JB, et al. Principles for evidence-based drug formulary policy. J Gen Intern Med 2005;20:964-8.

40. King MA, Roberts MS. The influence of the Pharmaceutical Benefits Scheme (PBS) on inappropriate prescribing in Australian nursing homes. Pharm World Sci 2007;29:39-42

Correspondence to: Dr. Irfan A. Dhalla, Department of Medicine, St. Michael's Hospital, 30 Bond St., Toronto ON M5B 1W8;

dhallai@smh.toronto.on.ca 